\title{
The incremental stimulus intensity effect and habituation of autonomic responses in man
}

\author{
J. G. O'GORMAN and R. D. JAMIESON \\ University of New England, Armidale, N.S.W. 2351, Australia
}

\begin{abstract}
Two experiments are reported on the effectiveness of a gradually increasing series of stimulus intensities for reducing autonomic responsiveness to a high-intensity stimulus. In the first, frequency of electrodermal (EDR) responding, but not of finger blood volume responding, to a $100-\mathrm{dB}$ (re .0002 dyne $/ \mathrm{cm}^{2}$ ) white noise was found to be significantly lower, $t(18)=2.35, p<.05$, if subjects were previously exposed to a range of intensities from 80 to $100 \mathrm{~dB}$ in ascending order than if exposure consisted only of repeated presentation of the $100-\mathrm{dB}$ stimulus itself. In the second experiment, magnitude of the intensity increment was shown to be more critical than the amount of training at each increment in a gradually increasing series of stimulus intensities for reducing EDR magnitude, $F(1,36)=$ $5.36, p<.05$, but not magnitude of the heart-rate response to a white noise of $100 \mathrm{~dB}$. Implications of these data for theories of the habituation process proposed by Sokolov and Groves and Thompson were discussed.
\end{abstract}

Studies of behavioral startle to white noise in the rat (Davis \& Wagner, 1969) and of hindlimb flexion to electric shock in the acute spinal cat (Groves \& Thompson, 1970) indicate that habituation to a stimulus of high intensity is greater when training consists of exposure to a series of stimuli which gradually increase in intensity than when training involves repeated presentation of the high-intensity stimulus itself. Groves and Thompson (1970) termed this the incremental stimulus intensity effect and proposed it as a general parameter of habituation, though generality of the effect beyond behavioral responses in infrahumans was not demonstrated. Generality of the effect cannot be assumed, since File (1973) has pointed to differences in the effects of stimulus intensity on long-term and short-term habituation. The present study examined the incremental stimulus intensity effect for habituation of human autonomic responses, which, unlike behavioral startle and hindlimb flexion to shock, habituate rapidly.

Two questions raised by previous work were posed about the effect. The first concerns the correlation between changes in phasic responsiveness and arousal level of the subject. The efficacy of an incremental series was argued by both Davis and Wagner (1969) and Groves and Thompson (1970) to be due to a change in arousal state of the individual. Although Groves and Thompson (1970) cited several studies indicating the influence of state on habituation, no evidence was adduced to support the hypothesis that

The research reported in this paper was supported by a grant to the first author from the Australian Research Grants Committee. Requests for reprints should be addressed to J. G. O'Gorman, Department of Psychology, University of New England, Armidale. Australia. 2351. R. D. Jamieson is now at the University of Aston in Birmingham, England. the incremental stimulus intensity effect was a result of concomitant changes in arousal level. Recording of resting level of skin conductance in the first experiment reported here and of skin conductance and heart rate in the second permitted a check on the hypothesis, since these are both common indices of arousal. The second question concerns the characteristics of an incremental series which determine the effect. Although the use of appropriate controls in previous studies indicated that the incremental nature of the series was critical for the effect, the importance of other aspects of the stimulus series, such as magnitude of the increment and number of presentations of each increment, has yet to be determined. Davis and Wagner (1969) interpreted their data as suggesting that magnitude of the increment is a more important characteristic than total amount of training, but no systematic study of the question was made. In the second experiment reported here, amount of training was compared with magnitude of increment to examine the importance of each factor.

\section{EXPERIMENT I}

The purpose of the first experiment was to compare the effectiveness of an incremental series of exposures with a series of exposures at constant intensity for reducing the frequency of electrodermal (EDR) and finger blood volume (FBV) responses to a white noise of $100 \mathrm{~dB}$ (re $.0002 \mathrm{dyne} / \mathrm{cm}^{2}$ ). The incremental series began with a stimulus of $80 \mathrm{~dB}$, which is below the threshold of aversiveness for white noise (Sullivan, Warren, \& Dabice, 1970), and increased in intensity by $5 \mathrm{~dB}$ every five trials up to $100 \mathrm{~dB}$. Two independent groups of subjects were employed, one for each condition. Over the last block of five trials, 
both groups were administered a $100-\mathrm{dB}$ noise, and comparison of the groups over this trial block permitted test for the effect.

\section{Method}

Subjects. Twenty males, ranging in age from 18 to 35 years, volunteered for the study. Fourteen were graduate students and six were technicians, and they were allocated in equal numbers to the two groups.

Apparatus. The habituating stimulus was a white noise of $1 \mathrm{sec}$ duration. which was presented at regular intervals of $40 \mathrm{sec}$. Duration and interstimulus interval were controlled by a tape programmer built in the department's workshop. Intensity of the noise was controlled by a Hewlett-Packard dc attenuator, Type $350 \mathrm{D}$. operated manually by the experimenter, and was measured using a Dawe sound level meter (Type 1419c) at the Akai stereo headset through which the stimulus was presented to the subjects.

An E \& $M$ Instrument Co. GSR preamplifier was used to record $\mathrm{EDR}$ from $\mathrm{Ag} / \mathrm{Ag} \mathrm{Cl}$ electrodes constructed according to the specitications of Venables and Martin (1967, p. 96). The electrodes were taped to the palm of the subject's left hand and a $.05 \mathrm{~N} \mathrm{NaCl}$ solution in an aqueous cream base-was used as the electrolyte. The FBV response was recorded using an $E \& M$ photoelectric dc transducer which was attached to the middle finger of the subject's right hand. One channel of an E \& M Physiograph 4 recorded $E D R$, and a second the FBV response. Stimulus onset was recorded on the event marker.

Procedure. Each subject was tested individually in a quiet. semidarkened laboratory while seated in an armchair. The experimenter monitored the study from an adjoining room which housed the stimulus equipment and recording apparatus. On arrival at the laboratory, the subject was told that the experiment was concerned with studying the physiological correlates of relaxation and that a moderately loud stimulus would be presented from time to time through the headphones. No specitic information was provided about the nature of the stimulus. Following attachment of the electrodes and a 10-15-min resting period, the first stimulus appropriate to the condition under test was presented. A total of 25 trials were run with each subject. For subjects in the constant-intensity group. these involved repeated presentation of the $100-\mathrm{dB}$ noise. For subjects in the incremental group, the trials involved tive presentations of $80-, 85-, 90-, 95-$, and $1(1)-\mathrm{dB}$ noise. in ascending order. At the end of the series, a postexperimental enquiry was conducted and any questions answered. The purpose of the enquiry was to determine if the subject was aware of any aspects of regularity in stimulus presentation.

Scoring. The EDR was quantified as the difference between conductance level immediately prior to stimulus onset (EDL) and the maximum level reached within $5 \mathrm{sec}$ following stimulus onset. Response was recorded in resistance units and then transformed to conductance. Response amplitudes of less than .005 micromhos were scored as zero. The FBV response was scored using a procedure similar to that described by Ginsberg and Furedy (1974), and involved measuring the shift in millimeters in the midpoint position of the FBV trace occurring within 2 to $8 \mathrm{sec}$ after stimulus onset. Shifts of less than $5 \mathrm{~mm}$ were scored as zero.

\section{Results and Discussion}

While all subjects in the incremental series were aware of an increase in intensity during the series, none could specify how many changes were involved or when they occurred.

Figure 1 presents the mean number of EDR and FBV responses for each block of five trials. For EDR, there is virtually no difference in responsiveness for subjects under the two conditions over the first block of trials, but thereafter, while subjects under the incremental series show a general decrease in responsiveness, subjects under the constant series show little drop. Over the last block of trials, the test block, in which both groups received the 100-dB noise, the mean number of EDRs for subjects under the incremental series was significantly lower than that for subjects under the constant series, $t(18)=$ 2.35, $\mathrm{p}<.05$. Thus, for EDR, the data confirm the incremental stimulus intensity effect.

The results with the FBV index failed to demonstrate the effect. Both groups showed far less decrease in responsiveness during the habituation series, and while the mean number of FBV responses for subjects under the incremental series was lower for the test block than that for subjects under the constant series, the difference was not statistically significant, $\mathrm{t}(18)=1.13, \mathrm{p}>.05$. Failure to demonstrate the effect with this index may be due to unsuitability of the schedule employed to produce habituation. Further training at each intensity level before proceeding to the next may be required to produce the effect with the FBV response. The literature on plethysmographic responses indicates, however, that response decrement with repeated stimulation is not as invariant as it is with the electrodermal response (e.g., Ginsberg \& Furedy, 1974).

A repeated measures analysis of variance on the EDL data for the points immediately prior to Stimuli $1,6,11,16$, and 21 indicated a significant effect for trials, $F(4,36)=5.22, p<.05$, but no significant effect for conditions or the interaction of conditions and trials, Fs $<1$ in both cases. Thus, while there was a decrease in EDL during both series, there was no significant difference in the rate of decrease between the two series. That is, differential phasic responsiveness was not associated with differential change in tonic level. While a correlation between phasic and tonic changes would not have provided unequivocal support for the hypothesis that the efficacy of an incremental series is dependent for its effects on the state of the organism, the failure to observe a correlation contradicts the hypothesis. The efficacy of an incremental series can be demonstrated without a change in state, at least as far as this construct was operationalized in the present experiment.

\section{EXPERIMENT II}

The purpose of the second experiment was to compare the importance of changes in the magnitude of the intensity increment used in :an incremental series with the amount of training at each increment, the training schedule, for reducing responsiveness. 
Figure 1. Mean frequency of response over trial blocks for electodermal (EDR) and finger blood volume (FBV) responses.

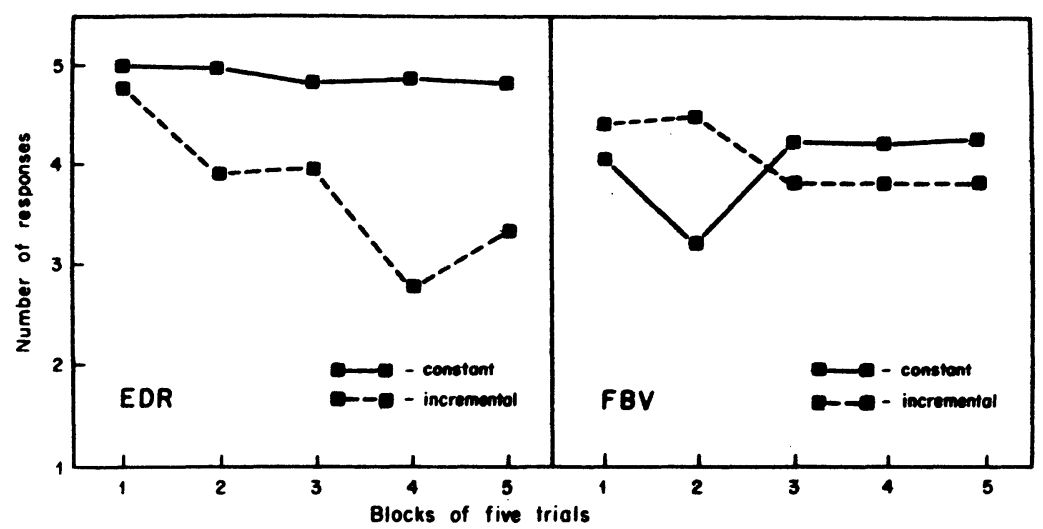

\section{Results and Discussion}

All subjects were aware that stimulus intensity increased during the series, but none were able to specify the number of changes or the schedule on which the changes were made.

Table 1 presents EDL data immediately prior to the first training stimulus and the test stimulus. A 2 by 2 by 2 (Increments by Training Schedule by Trials) analysis of variance with repeated measures on the last factor indicated a significant interaction between increments and trials, $F(1,36)=14.38, p<.01$, and a significant three-way interaction, $F(1,36)=5.05$, $p<.05$. No other main effects or interactions proved significant. Tests of simple main effects for the trials factor indicated that only the decrease in EDL from pretraining to pretest for the group receiving five presentations of the 2-dB increase was significant, $F(1,18)=18.07, p<.01$. Comparisons in EDL between pretraining and pretest for groups receiving five presentations of the 5-dB increment, $F(1,18)=$ $3.71, p>.05$, two presentations of the $5-\mathrm{dB}$ increment, $F(1,18)=0.40, p>.05$, and two presentations of the 2-dB increment, $F(1,18)=21$, $\mathrm{p}>.05$, were not significant.

Mean EDR magnitude on the test trial under the four conditions is presented in Table 2. Magnitude of EDR was lower under conditions in which a 2-dB increment was employed. A 2 by 2 analysis of variance indicated a significant main effect for increments, $F(1,36)=5.36, p<.05$, but no main effect for training schedule, $F(1,36)=1.64, p>.05$, and no significant interaction, $\mathrm{F}<1$.

Table 1

Means and Standard Deviations of EDL (in micromhos $x$ 1000) and HRL (in seconds) for Each Experimental Group Prior to the First Training Stimulus and the Test Stimulus

\begin{tabular}{|c|c|c|c|c|c|c|c|c|}
\hline \multirow{2}{*}{$\begin{array}{l}\text { Experimental } \\
\text { Group }\end{array}$} & \multicolumn{4}{|c|}{ EDL } & \multicolumn{4}{|c|}{ HRL } \\
\hline & \multicolumn{2}{|c|}{ Pretraining } & \multicolumn{2}{|c|}{ Pretest } & \multicolumn{2}{|c|}{ Pretraining } & \multicolumn{2}{|c|}{ Pretest } \\
\hline $\begin{array}{l}5 \mathrm{~dB} \times 5 \text { Trials } \\
5 \mathrm{~dB} \times 2 \text { Trials } \\
2 \mathrm{~dB} \times 5 \text { Trials } \\
2 \mathrm{~dB} \times 2 \text { Trials }\end{array}$ & $\begin{array}{l}148.8 \\
153.3 \\
154.2 \\
149.1\end{array}$ & $\begin{array}{l}(48.3) \\
(40.4) \\
(44.7) \\
(50.7)\end{array}$ & $\begin{array}{r}175.8 \\
142.2 \\
94.6 \\
135.7\end{array}$ & $\begin{array}{l}(82.6) \\
(63.0) \\
(50.4) \\
(62.5)\end{array}$ & $\begin{array}{l}.72 \\
.79 \\
.72 \\
.81\end{array}$ & $\begin{array}{l}(.12) \\
(.11) \\
(.10) \\
(.14)\end{array}$ & $\begin{array}{l}.78 \\
.77 \\
.80 \\
.79\end{array}$ & $\begin{array}{l}(.09) \\
(.12) \\
(.12) \\
(.12)\end{array}$ \\
\hline
\end{tabular}


Table 2

Means and Standard Deviations of HRR (in seconds) and EDR (in micromhos $x$ 1000) on Test Trial for the Four Experimental Groups

\begin{tabular}{lcr}
\hline $\begin{array}{l}\text { Experimental } \\
\text { Group }\end{array}$ & HRR & \multicolumn{1}{c}{ EDR } \\
\hline $5 \mathrm{~dB} \times 5$ Trials & $.12(.07)$ & $9.2(10.5)$ \\
$5 \mathrm{~dB} \times 2$ Trials & $.12(.08)$ & $11.3(11.8)$ \\
$2 \mathrm{~dB} \times 5$ Trials & $.12(.09)$ & $1.2(2.0)$ \\
$2 \mathrm{~dB} \times 2$ Trials & $.14(.04)$ & $6.3(5.6)$ \\
\hline
\end{tabular}

The pattern of results with the electrodermal data support the conclusion of Davis and Wagner that increment size is more important than amount of training in producing response decrement with an incremental series, but are not wholly consistent with their view that this depends on concomitant changes in state. Whereas magnitude of the increment alone was responsible for decreased phasic responsiveness, both magnitude of the increment and amount of training were important in determining tonic level.

The cardiac response data (see Tables 1 and 2), by comparison, provided no support for the incremental stimulus intensity effect. A 2 by 2 by 2 analysis of variance with repeated measures on the last factor performed on HRL yielded $F$ values greater than unity only for the training schedule factor, $F(1,36)=2.33$, $\mathrm{p}>.05$, and the trials factor, $F(1,36)=2.91$, $\mathrm{p}>.05$. A 2 by 2 analysis of variance on the magnitude of HRR to the $100-\mathrm{dB}$ stimulus resulted in no significant main effects or interactions, Fs $<1$ in all cases.

\section{GENERAL DISCUSSION}

The present experiments extend previous findings on the incremental stimulus intensity effect with behavioral responses in infrahumans to habituation of the electrodermal response in man. Whether the effect can be demonstrated with autonomic components other than EDR remains uncertain. The lack of success experienced in the present study with cardiovascular components may be a result of the particular stimulus schedules employed, and it would be premature to conclude on the basis of the present data that the incremental stimulus intensity effect does not hold for systems other than EDR.

Interpretation of the data from the present study raises difficulties for major theories of the habituation process. As Groves and Thompson (1970) pointed out, an expectancy theory of habituation such as that advanced by Sokolov (1960) leads to the prediction that a series of stimuli at constant intensity will produce greater habituation than a series of varying intensities, since the process of habituation is postulated to depend on the neural coding of the parameters of stimulation and the fewer the variations the more rapid the process. In Experiment I, responsiveness over the last trial block, according to
Sokolov's theory, should have been greater in the incremental series because of the novelty of the stimulus. In Experiment II, the theory would predict an effect for amount of training, since the precision of the neuronal model responsible for habituation in the theory should be increased with stimulus presentation. These data are particularly pertinent to a criticism of Sokolov's theory since they arise from a paradigm, habituation of human autonomic responses, on which the theory was based, whereas previous studies have dealt with habituation of behavioral responses in infrahumans.

It may be objected that Sokolov's theory was developed to account for habituation to stimuli within the moderate range of intensity, stimuli which elicit orienting responses in Sokolov's taxonomy, and not to account for habituation to stimuli of high intensity, those which elicit so-called defensive responses. The importance of this objection can be evaluated by examining the dependence of the incremental stimulus intensity effect on the intensity of the test stimulus, with concurrent recording of vascular responsiveness in the finger and forehead permitting the identification of the two types of response. The objection loses some force, however, when the data of Experiment II are considered, since here EDR amplitude was determined by the magnitude of the increment and this would be predicted by Sokolov's neural matching theory. Thus, the theory cannot be totally rejected by the present results.

As well as questioning Sokolov's account of habituation, the data are not satisfactory from the point of view of the Groves and Thompson theory. In essence, the theory proposes that response amplitude as a result of stimulus repetition is the outcome of a decremental process in the specific $S-R$ system activated by the stimulus and an incremental process in the general arousal state of the organism. The incremental stimulus intensity effect, in these terms, is the result of smaller increments in arousal state. There was no correspondence, however, in Experiment I between electrodermal tonic level and phasic responsiveness consistent with the Groves and Thompson position. In Experiment II, there was no systematic change in $H R \mathbf{L}$, a usually robust index of arousal, and yet differences in EDR predicted by the Groves and Thompson theory were obtained. This may mean that the state construct in their theory was not validly measured in the present experiments, though it is difficult to suggest alternative indices which would be more appropriate, or it may mean that, as Lacey (1967) argued, a concept of general arousal level is overly simple when applied to autonomic functioning.

The data of the present study, while demonstrating the incremental stimulus intensity effect for habituation of the electrodermal response in man, cannot be wholly explained in terms of current theories of habituation. 


\section{REFERENCES}

DAvis, M., \& WAGNeR, A. R. Habituation of startle response under incremental sequence of stimulus intensities. Journal of Comparative and Physiological Psychology, 1969, 67, 486-492.

File, S. Effects of stimulus duration and intensity on the rate and speciticity of behavioural habituation. Quarterly Journal of Experimental Psychology, 1973, 25, 96-103.

Ginsberg, S., \& Furedy, J. J. Stimulus repetition, change, and assessments of sensitivities of and relationships among an electrodermal and two plethysmographic components of the orienting reaction. Psychophysiology, 1974, 11, 35-43.

Groves, P. M., \& Thompson, R. F. Habituation: A dualprocess theory. Psychological Review, 1970, 77, 419-450.

LACEY, J. I. Somatic response patterning and stress: Some revisions of activation theory. In M. H. Appley \& R. Trumbull (Eds.), Psychological stress: Issues in research. New York: Appleton-Century-Crofts, 1967.
Lang, P. J., \& Hnatiow, M. Stimulus repetition and the heart rate response. Journal of Comparative and Physiological Psychology, 1962, 55, 781-785.

Soxolov, E. N. Neuronal models and the orienting reflex. In M. A. Brazier (Ed.), The central nervous system and behavior. New York: Josiah Macy, Jr., Foundation, 1960.

Sullivan, R., Warren, R., \& Dabice, M. Minimal aversion thresholds for white noise: Adaptation. American Journal of Psychology, 1970, 83, 613-620.

Venables, P. H., \& MARtin, J. Manual of psychophysiological methods. Amsterdam: North-Holland, 1967.

(Received for publication May 12, 1975; accepted June 10, 1975.) 